\title{
VORTEX ENTERPRISE: DECENTRALIZED VIRTUAL REALITY BLOCKCHAIN-BASED PLATFORM
}

\author{
UDC (336.744:004.946)
}

\author{
Aleksandar Jovanović, Aleksandar Milosavljević \\ University of Niš, Faculty of Electronic Engineering, Department of Computer Science, \\ Niš, Republic of Serbia
}

\begin{abstract}
Virtual Reality (VR) has been used in industries since the 1960s. However, the recent development of hardware and software has made it possible for VR technologies to become widely adopted by the mass market with the highest level of immersion and affordable price. However, building platforms for VR technologies will require many active users and their engagement in project development. Those activities will include platform activities, content creation, social media marketing, processing power, and storage. For that purpose, we introduce VoRtex enterprise highlevel software architecture and decentralized blockchain solution. Introduced VoRtex enterprise high-level software architecture will be used to develop a blockchain-based virtual world $(V W)$ platform with the VR support. The goal of the VW platform is to support the development of education, certification and massive open online courses (MOOC). A blockchain component inside the VW platform will be used for engaging users in project development through collaboration and content sharing. Active users that promote platform content will get rewards for contributions using cryptocurrency. Also, we will showcase this feature on the VoRtex platform prototype where the user earns cryptocurrencies for activity inside the VW. In the end, we will evaluate the proposed solution.
\end{abstract}

Key words: virtual world, virtual reality, enterprise, education, blockchain

\section{INTRODUCTION}

The virtual reality (VR) technology has been in development since the 1960s. However, with the advent of ultra-high-definition (UHD) screens, increased velocity of the GPU and lower cost, it becomes available for the average consumer to own VR headmounted devices (HMDs). VR is used to provide an immersive experience inside digital environments like gaming, 3D asset store, real estate, or training simulations. Most

Received December 03, 2018

Corresponding author: Aleksandar Jovanović

Faculty of Electronic Engineering, Aleksandra Medvedeva 14, 18000 Niš, Republic of Serbia

E-mail: aleksandarjovanovic@elfak.ni.ac.rs 
modern VR HMD devices are Oculus Rift, HTC Vive, and Google Cardboard. HMD headsets have the potential to improve processes and effectiveness in the enterprise by reducing the need for physical premises of consumer training and engagement programs with a better understanding of consumer behavior. Also, because of technical limitations, VR content creator should follow the best practices and standards regarding user experience design and software optimization [1]. The VR user interface is becoming increasingly popular in the virtual world (VW) platforms. VWs use 3D-generated virtual spaces that contain 3D models, 2D image or a video stream in which remote users can collaborate. VWs are the representation of 3D virtual space where users as avatars can interact with each other. Also, VW can be used interchangeably. A vital feature of the VW is a persistent area because other avatars continue communicating after we log out from the virtual world. Nevelsteen [2] provides a detailed definition of a virtual world, with all underlying terms defined based on new emerging technologies. The social VWs differ from the Massive Multiplayer Online Game (MMOG) concept because of active socialization between users. Also, an interaction in a social VW can achieve social realism. However, emerging social VW platforms are experimenting with democratizing access and ownership of content using decentralized systems like blockchain. The blockchain concept can be adapted in different sectors and for that reason, we propose a solution that can be used in the educational sector with a goal to provide security benefits and transparency between users based on advantages from distributed systems.

In this paper, we propose an innovative software architecture that will leverage blockchain for engaging users in the development of the VW platform. The article was organized in the following way. Firstly, we will review related work regarding VW and blockchain. Also, we will introduce scientific and commercial projects in these fields. Secondly, we will describe the concept and purpose of the blockchain-based solution in VWs. Thirdly, we will introduce VoRtex enterprise decentralized blockchain-based software architecture to support the development of education, certification and massive open online courses (MOOC) [3]. Also, we will describe the components and technologies that will supply our decentralized project. Afterwards, we will showcase a practical example inside VoRtex platform using cryptocurrency to engage the user in session activities. Then, we will provide a feature table that contains a comparison between similar solutions and VoRtex platform. In the end, we will provide guidelines for further improvements.

\section{RELATED WORK}

Current issues that are common in VW and MMOG video games are content monetization, scalability, asset creation, and verifiability of virtual objects. One solution to these issues is the adaptation of a decentralized system based on blockchain. Theoretically, decentralized systems like blockchain can provide instant and immutable transmission of data to improve the performance of VW platforms. Also, merging blockchain and virtual reality (B-VR) creates a new technology field that included peerto-peer (P2P) network, blockchain integration, cryptocurrency user engagement, and virtual storage scalability. B-VR platforms represent VW with support for VR user interface over HMDs. Also, the benefits of blockchain technology have been mentioned 
in different solutions [4]. We will describe the different use cases and research papers regarding P2P network, VR and VW platforms, and blockchain technologies in education.

Researchers succeeded in using VR technology for collaborative applications, such as real-world annotations, and mobile telepresence where users were at the remote location. However, studies discover few limitations. For instance, each virtual environment had to be recreated from scratch, with the cost of additional processing power and time. Also, the presented solution worked only with one virtual space per session without the option to relocate, although studies indicate that remote collaboration can benefit from multispace and multi-viewpoint interactions in a spatial context like a video stream with geospatial data. However, introducing such mapping functionality poses an architectural challenge: a public system that stores data should be resilient, in case of many requests, and immutable, to prevent alteration of previously archived spaces by third parties. These issues were partially addressed in commercial social VR networks [5].

Bektur Ryskeldiev et al. [6] propose a model for decentralized virtual reality space storage and distribution. The proposed solution combines several essential concepts: remote collaboration through mixed reality (MR, blending between real and VWs) and decentralized blockchain-based storage. VW does not need to ensure the validity of user transactions; the authors decide to move the data into the blockchain. Transferring data to blockchain provide advantages: unique identifiers (ID) for each space using generated hash codes; Data is immutable because changing the data payload would volatile the chain. Also, all data is stored in a plain text JSON array, and all session participants are connected in a P2P network using real-time web communication (WebRTC) protocol. For newly created space system generate a block containing the URL to the photospheric image, geo-location, and creation time. Afterwards, blockchain is updated, and users download a valid chain with newly added blocks where they can join active sessions and collaborate in real-time using audio, video, and 3D annotations in virtual space. Also, users can see all available virtual spaces sorted by locations and select them based on geographical coordinates of the area they are currently located.

P2P architectures have been proposed as a scalable solution for supporting massively multi-player applications like VWs or video games. However, the primary challenge for P2P designs consists of providing each avatar with updated information about which other avatars are its neighbors. This problem is known as an awareness problem. Rueda et al. [7] performed using well-known performance metrics in distributed systems. The evaluation shows that only a single method provides full awareness and best performance to avatars. The results also show that the COVER method offers the best system performance regarding well-known metrics like latency and throughput.

The neighbor table or distributed hash table (DHT) is used to choose the data supplier for data-dispatching services in distributed virtual environments based on P2P networks. Mingfei Wang et al. [8] summarized the common social behaviors of avatars and extracted their characteristics and then proposed an interest-similarity measuring algorithm to divide the node into several clusters. They measured the cluster stability regarding interest entropy while constructing a stable neighbor mesh for each node in a cluster. Also, they conducted extensive simulation experiments that simulate avatar behaviors in an MMOG. The results show that the proposed mechanism achieved a substantial alleviation of neighbor churn and reduced information exchange, which improves the transmission efficiency in distributed virtual environments. Mobile agents (MA) have been established as a robust programming 
method for heterogeneous networking and computing environments. Theodore Kotsilieris et al. [9] propose two distributed VW architectures, namely loose and tight. In parallel, they presented a relevant implementation scheme along with experimental results that prove the performance enhancements achieved against the classic client-server model. The proposed architecture is based on the MA technology. They verified that the performance of the MAbased approach exceeds that of the classical client-server model in different load conditions.

Regarding applying blockchain in education, there are solutions for verifying student profiles based on academic achievements and higher education credit platforms. The European Union has also shown interest in using blockchain for educational by analyzing the technology and possible risks for personal and academic training. Grech and Camilleri [10] conclude that blockchain usage in the education sector has not yet been sufficiently developed, but has a promising future. Antonio Bartolome et al. [11] address the fundaments and developments of blockchain technology in the specific education sector. Turkanović et al. [12] propose an EduCTX solution that represents a blockchain-based higher education credit platform. The EduCTX goal is to create a transparent and advanced form of higher education systems. The EduCTX prototype is based on the open-source Ark blockchain platform [13]. Based on a P2P network, EduCTX process and manage ECTX tokens, which represent credits that students get for completed courses. Another solution is the Disciplina project by TeachMePlease [14], a solution for storing educational records in the blockchain network. Disciplina has dedicated blockchains platform that is developed for the demands of the educational and recruiting fields. Disciplina blockchain in comparison to ETH, NEO (low-cost transactions) and EOS (POS consensus) platforms additionally provides a web of trust to establish the authenticity and private chain. The goal of the platform is to create a practical algorithm for students search by fields of expertise and to develop a monetization system for data stored by the higher educational institutions. Also, the platform provides a simple interface between students, teachers, and human resource managers.

Listed papers are focused on the virtual environment, generating data, and performance between nodes in the distributed system. However, they are not focused on blockchain-based virtual world platforms. In the next chapters, we will introduce the blockchain and B-VR virtual world platform.

\section{BLOCKCHAIN TECHNOLOGY REVIEW}

Shared ledger technology, commonly called Blockchain, provides a new approach to enable collaborations among people and organizations. Shared Ledger is DDBMS that leverage cryptography to offer a decentralized multi-version concurrency control mechanism and consensus to achieve overall system reliability in a distributed network [15]. The DDBMS synchronizes all the data periodically, and in cases where multiple users access the same data, ensures that modifications (update and delete) performed on the data at one location will automatically reflect in the data stored elsewhere. The blockchain can be programmed to record sensitive data. It uses public infrastructure (PKI) asymmetric cryptography for establishing security, where users get two keys: one for encryption and second for decryption. All information on a blockchain network exists as a shared and decentralized database, where all records are public and verifiable. Blockchain provides a productive, secure, and transparent platform on which we can create a decentralized network. Also, blockchain is a digitized, decentralized public ledger of cryptocurrency 
transactions. Necessarily, each block is an individual statement, and completed blocks are added in the chronological order allowing users to keep track of the operations without the need for a central server. For example, Bitcoin (BTC) [16] eliminates the need for a central server to process transactions. Users in blockchain structures are responsible for validating operations inside the network. For blockchain application development, we can choose between public (BTC, ETH, DASH, and NEO) or permissioned (Ripple, Eris, and Hyperledger Fabric v0.6) blockchain for our solution. Each blockchain platform has different goals and features for developing blockchain network. The public blockchain is permissionless and open without exception to every user in the network. Users can download the entire history of the blockchain on their device, store information, or create SMs. Permissioned blockchains give control of the network to their owners (consortium or centralized to one organization). Permissioned blockchains have a high level of flexibility and can adjust to different access levels of public exposure, user authorization, and execute blockchain as a stand-alone or integrated into the existing system. While public blockchains act in a trustless environment, permissioned blockchains require a higher level of trust among their members. Permissioned blockchain benefits from higher speeds and ensures internal security.

The smart contracts (SM) [17] or automated computer programs manage the collaborations in the blockchain. A Blockchain DApp uses SMs to execute commands and retrieve information from Blockchain. Therefore, SMs can include contractual arrangement, the actual execution of the contracts, and the governance of the preconditions required for contractual obligations. All transaction operations must be deterministic. Similarly, you cannot have SMs that work in two different ways in two different machines.

Currently, decentralized blockchain applications have few options to store data. Secondly, the immutability is the strength of blockchain with high robustness, but with drawbacks for a data storage. Content distribution through a P2P network has two main issues. The first involves downloading speed because retrieving a file from a DHT or distributed P2P storage system has traditionally been too slow. The second issue requires availability or ensuring that the content is sufficiently distributed around the network without loss. For example, the InterPlanetary File System (IPFS) is based on the BitTorrent protocol and Distributed Hash Table. This technology allows sharing data on other computers, creating the global file system with addressable content where preferred files can be downloaded very quickly using the BitTorrent protocol. However, it also has drawbacks that require that computer with shared data should stay online. There are decentralized cloud file storages that eliminate some of IPFS drawbacks. In this case, the content is hosted on computers which offer their hard drive space using pay-as-you-go services, so the user does not need to stay online.

Examples are Sia, Storj, ETH Swarm, etc. Due to strict transactional SQL databases cannot be genuinely distributed because of the CAP-theorem. To make a database distributed, we must sacrifice either consistency or availability. NoSQL databases, choose availability over consistency (eventual consistency) where all the database nodes in the network become consistent after some time. Examples are MongoDB, Apache Cassandra, RethinkDB and, etc. NoSQL solutions are fast, scalable, fault tolerant, but they are not Byzantine-proof (dependability of a fault-tolerant computer system), and the malicious node can destroy the database system. The BigChainDB solution claims to solve the data storage and speed problem with significant data capacity and fast transactions. 
Ethereum [18] platform goal is to create a solution for the development of distributed applications (DApps) it is widespread in blockchain-based solutions. ETH is an established project with a functional platform that uses the same protocol as BTC called Proof of Work (PoW). In Proof of Work protocol, computers on the network solve mathematical equations and winner acquire right to confirm transactions into the blockchain. The two drawbacks to the PoW protocol is $51 \%$ attack and the energy consumption for network security. Because of that, there is alternatively called Proof of Stake (PoS). PoS works similarly to PoW except that it uses token holders. Those who hold tokens (staking), will earn awards based on the number of tokens they held. Token sales mostly happen on ETH platform using the ERC20 standard [19]. ERC20 is a technical standard used for SM on the ETH blockchain for implementing tokens in blockchain-based solutions. Also, tokens will be compatible with any other client or wallets that use ERC20 standards. Beside ETH there are other platforms like Hyperledger Fabric (GoLang and Javascript), NEM, Neo (.NET, Java, Kotlin, Go, Python, etc.), $\mathrm{EOS}(\mathrm{C} / \mathrm{C}++)$, or Lisk (Javascript) that are used for DAPPs and SM development.

Based on ETH security issues, there is alternatively named NEO. NEO utilizes blockchain and digital identity to digitize assets. NEO uses a dBFT (Delegated Byzantine Fault Tolerance) protocol, which is a modification of the PoS protocol based on listed issues regarding security there is an alternative for ETH named NEO. EOS is a blockchain and an SM platform with no transaction fees. The EOS platform uses a delegated proof of stake (DPoS) consensus mechanism, which makes the network centralized in exchange for a scalable and faster system. Both solutions are public blockchains. An enterprise permissioned blockchain network Hyperledger Fabric (or Fabric) supports a high-reliability, shared, trusted, privacy-preserving, a non-reputable data repository. Also, it shares many similarities with traditional client server-based systems. The Fabric has a modular architecture and provides flexibility through development process. For example, it is not possible in ETH to have a transaction visible to a few peers and not visible to others. Chaincode is an SM that represents a piece of code deployed to Fabric for enabling interactions between peers and the shared ledger. A chaincode can be installed on a blockchain by executing a deploy transaction while invokes transactions launch a chaincode execution. Distributed peers will validate the incoming requests and propose transactions by running the chaincode. The platform uses a lot of accessible database and data streaming technologies to generate higher performance. The data is read directly from the state database, which can be based on CouchDB.

The most important reason for choosing the right language for SM development are security, resource management, performance, and isolation from non-deterministic elements. Solidity is the most mature language for SM development on ETH platform. Solidity is a loosely-typed language with a similar syntax to Javascript. Also, Solidity is more popular than $\mathrm{C}++$ or GoLang for DAPPs and SM development. The main reason why SM is developed using solidity is a deterministic feature of the language. For example, Go is not deterministic and could lead to fatal code behavior without strictly defined standard. 


\section{BLOCKCHAIN-BASED VIRTUAL WORLDS}

ICO (Initial Coin Offering) is an alternative fundraising process in which startups issue their crypto tokens, and sell them in exchange for BTC or ETH. The blockchainbased VW ICOs target artists, gamers, and online communities who are interested in making revenue through building and selling VR and 3D experiences. In this chapter, we will describe blockchain and VR based projects, which are: Decentraland, CEEK, The Deep, Ethernia, MARK.SPACE, Somnium Space, Staramba Spaces, Terra Virtua, VIBEHub, Virtual Universe, and other platforms. Listed B-VR projects are an excellent example of blockchain technologies being utilized outside of the finance sector. A centralized commerce system like Sansar and Second Life will not be described in this chapter. Mostly, all solutions are at an early stage of development or have a white paper to showcase future platform functionalities.

\section{A. CEEK}

CEEK VR is a distributor of immersive VR content that provides social and 3D experiences for multiple VR channels. Some of the VR experiences include 360 videos like music, charities, and sporting events. These events can be viewed using VR HMD devices while physically being in another location. Also, using remote access to event platform can solve issues regarding selling out ticked and enable an unlimited amount of virtual tickets. The CEEK distribution platform is compatible with all smartphones, Smart TVs, desktop, VR, AR, and MR devices. However, because of mainstream adoption of VR and cost of desktop and console VR device, CEEK offers low cost ready-to-use mobile VR headset. CEEK has its brand of cryptocurrency, also called CEEK. CEEK tokens are ERC20 compliant and let users used them for different experiences like voting, user engagement in contests, and purchasing virtual goods on the CEEK platform. Also, each virtual item has been assigned an Etherum address and protected using a unique cryptographic token signature. CEEK has a Celebrity Coin Cast that gives the entertainment industry access to mint custom coins and trades them within the platform. Using Celebrity Coin Cast, the artist can create an event ticket and provide a new revenue stream. Users can earn coins by creating digital content like custom skins, gift cards, curating events, creating quality content for education or promoting the platform on social media. Software developers can earn CEEK token by leveraging CEEKs tools, open APIs and multi-language SDKs, which allow users to create virtual worlds. For audio quality, CEEK has 4D sound technology with a dedicated digital signal processor (DSP) that combines haptic feedback and proprietary physics-based 3D audio with visual rendering. CEEK has an independent VW called CEEK CITY. CEEK CITY includes environments such as a theatre, sports stadium and more [20].

\section{B. Decentraland}

Decentraland is a VW open-source community-driven platform where users can access with VR HMD via the web browser. It uses distributed storage paired with blockchain, a continuous immutable ledger of transactions that ensure the delivery of a virtual space to users. Users of Decentraland can explore user-generated content or create their own experiences and resell them on the platform. Decentraland is created using A- 
Frame an open-source web framework for building VR experiences on web browsers. Decentraland relies on WebGL technology and supports the production of virtual objects in the development environment like Unity. Decentraland was constructed on the Ethereum blockchain with SMs and consensus algorithms on a decentralized P2P network that is used during the transactions between virtual properties and the monetizing immersive content and experiences. A blockchain is established in parallel, and it is used to manage land collaboratively. Providing available computer time on user devices, users can claim or transfer virtual property. Decentraland uses BitTorrent and Kademlia DHT networks for storing a magnet link for virtual properties. In comparison to other traditional VW platforms such as Sansar and High Fidelity, we can build VR-capable experiences at a much lower cost than in Decentraland on our dedicated PC devices [21].

\section{Vibehub}

Vibehub is a virtual reality and blockchain platform for creating virtual spaces in which a large variety of activities can be conducted, from marketplaces to business meetings. Vibehub provides 3D photo-realistic in-house holograms (Holoportation) technology for full body scanning of musicians and educators, and with the ability to place them fully observable in custom VR or AR environments. Similar principles of hologram technology, we can see in the body scanner solution based on photogrammetry with the ability to upload a scanned 3D object to XR (extended reality) environment. XR environment allows users to observe a photo-realistic representation of an avatar and immersive collaboration with other avatar holograms using HMDs for augmented, mixed or VR. Vibehub platform internal currency is the VIBE token (ERC20), which users can use to buy and sell experiences on the vibe marketplace, and there are no GAS fees charged for microtransactions due to their private off-chain nature. The VIBE token can be traded on numerous exchanges such as Binance, Ether Delta, or HitBTC cryptocurrency exchanges. Vibehub allows users to buy or sell virtual products, including live performances, music, and unique offerings from celebrities like lectures. As the platform is decentralized, the user can use a wallet service and social features such as profiles and postings on a trust less and immutable ledger that guarantees the integrity of the data. Also, The Vibehub allows users to monetize VR content like designs, models, scenes, music or videos [22].

In the section below will describe software architecture for VoRtex [23] educational platform and provide a software architecture estimation.

\section{VORTEX - BLOCKCHAIN-BASED VIRTUAL WORLD SOFTWARE ARCHITECTURE}

VoRtex is an educational platform with enterprise features and virtual industrial environments to employ training from different fields like automotive, healthcare and space. The motivation behind using blockchain in VoRtex platform comes with an idea to build a foundation of trust in the VW, enable open-source collaboration on virtual research and secure VW for education and experimentation. Also, it introduces features that are not implemented on other platforms and with the goal to build a better user experience with a focus on learning inside a VW. The proposed enterprise software architecture for blockchain-based VW projects represents an immersive Virtual Learning Environment (VLE) with the multi-users support where users can access VWs and 
collaborate with remote instructors. The proposed solution adopts a user-centric design and enables processing of data using the blockchain network, that allows data ownership and integrity. A back-end solution contains a decentralized blockchain-based model, where content is represented as blocks containing the required information (such as links to educational material or generated $3 \mathrm{D}$ content), and where data is synchronized between connected users. Afterwards, the content will be archived, recycled, and shared among different users. This VLE also includes data acquisition from virtual words and blockchain-based secure identity solution. Also, a persistent and continuously updated collection of virtual spaces could decrease the computational costs for VW applications and expand the available interactive scope. Proposed architecture should be adaptable for all XR technologies. Other components that represent the building blocks of our architecture will be described in the following chapter.

\section{A. Software architecture outline}

For a better understanding of the platform, we provide a high-level architecture, including other features of the system outside the blockchain component. Software architecture consists of the following building blocks (Fig. 1):

- VWs system (VWs produced by content creators)

- Avatars (human maker and customisation)

- Intelligent agents (virtual assistance and chatbots)

- SaaS and PaaS interface for cloud infrastructure

- Learning material (lectures or video tutorials)

- Data analysis system (tracking progress component)

- An access control system (biometric and blockchain based solution)

- Blockchain network (permissioned and permissionless)

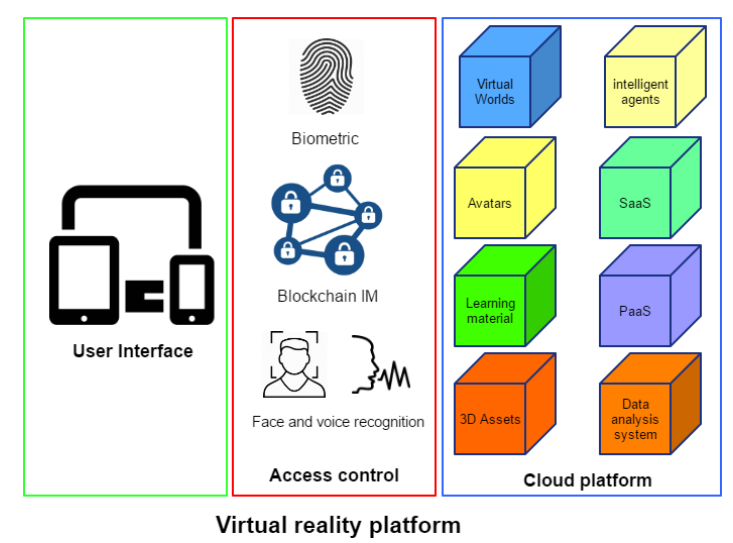

Fig. 1 VoRtex high-level component-based architecture

The VWs system should follow standards for content creation:

- Open space with a central area and clear boundaries in which users have the freedom to move and interact with objects and manipulate components, with immersive and gamification features like role-playing and teamwork collaboration. 
- The lesson has a unique name and direct access to the VLE.

- Each lesson should provide admin controls for teachers with functionality to manipulating lesson content.

- Each lesson should provide access to learning material for students.

- Allows users in an environment to communicate using adjustable audio levels based on the distance between users. This feature enables working in a small group where students may discuss privately and provide the immersive effect of the user sounding.

The blockchain network is used for storing data collected from client devices, and data providers, where hashed data is uploaded for integrity protection. Data access from the data provider, each of the data access requests should be processed to get permission from the data owner with a decentralized permission management protocol. A decentralized permission management protocol is a central part of the decentralized identity layer. We use public engagement layer for distributing platform VoRtex cryptocurrency using public engagement layer. Together, decentralized identity layer and public engagement layer construct blockchain system behind the platform (Fig. 2). The cloud database stores user-related data, data requests from the data provider, data access record and data access control policy. Also, once data leakage is detected, then the malicious entity can be identified. Using the data analysis system, individual users can contribute to future research, and valuable insights can be derived.

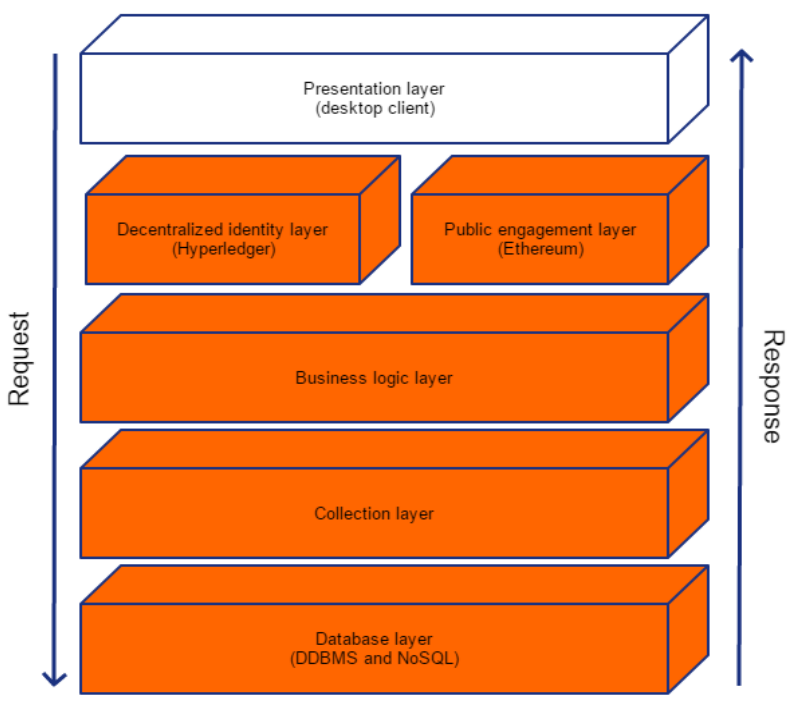

Fig. 2 VoRtex layered architecture

\section{$B$. Decentralized identity layer}

Don Tapscott and Alex Tapscott address issues regarding traditional higher education [24]. The challenge is to provide and maintain the privacy and security of stored digital data by academic institutions. When it comes to cybersecurity, colleges and universities are vulnerable as other organizations. Addressing its validity is another challenge. At a 
time when information is mutable, being able to verify a job prospect's claim is becoming valuable to companies or employers. Because of that, employers requiring to see official college transcripts and issuing transcripts to universities can charge transaction fees. Based on the described issues, we design a decentralized identity layer as a part of the VoRtex blockchain network.

A decentralized identity layer contains a decentralized permission management protocol for protecting user ownership of personal data. To preserve the integrity of data, within each record, proof of integrity and validation are permanently retrievable from the cloud database and is anchored to the blockchain network. The proposed decentralized identity layer in software architecture (Fig. 3) stores data from a university that student attends (student projects or exams) or MOOC progress from other learning platforms. Data providers such as teachers or course authors can send the user to perform the test. The test can be uploaded to the blockchain network for data sharing with other data providers under the user's permission. Also, each request and access activity should be stored on the blockchain network for auditing. When it comes to data processing and potential issues regarding safety and security, a university will be more comfortable with the permissioned blockchain solution. Also, developing a solution for higher education will require techniques widespread in enterprise software development (enterprise design patterns, web services, and workflow process). The system is based on Hyperledger Fabric and used for the following purposes:

- Data collected from both client devices and data providers.

- Personal data access from the data provider, each of the data access requests should be processed to get permission from the data owner with a decentralized permission management protocol.

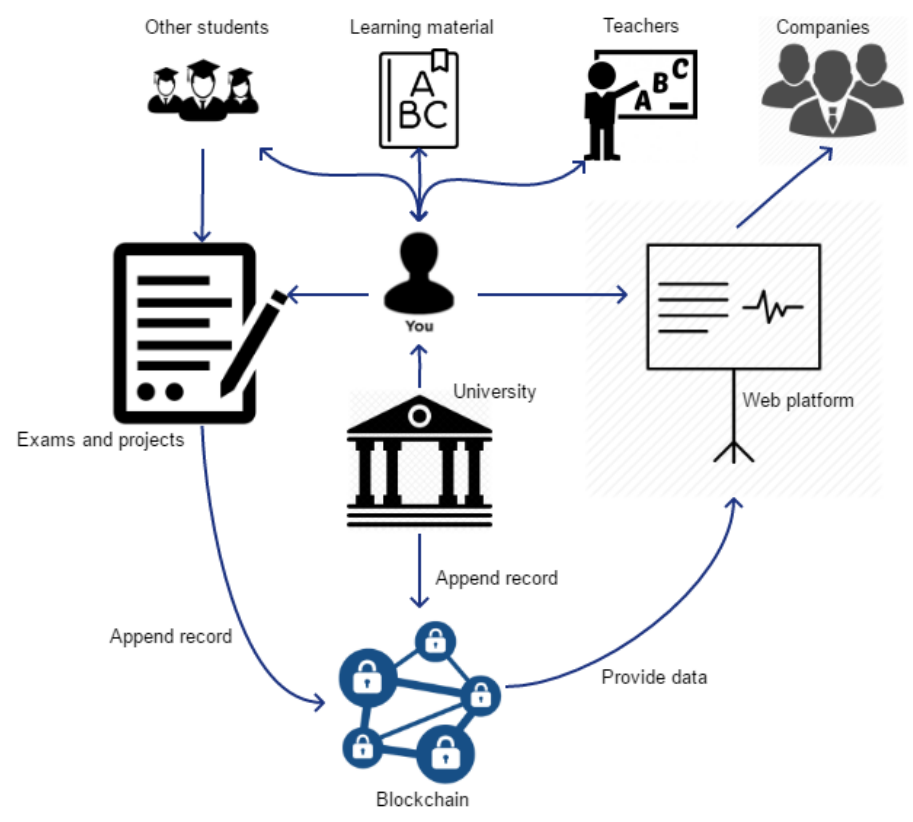

Fig. 3 Decentralized identity layer 


\section{Public engagement layer}

The idea of content creation and user engagement are introduced on established VW platforms. The notion of the monetizing virtual real estate first appears in Second Life to enable users to get revenue from digital content by creating high-quality content and retain the copyright for these virtual goods. However, it is difficult to improve the companyowned platform that generates values from user activities. Mostly, this kind of issues can be solved using the blockchain as distributed databases. The public engagement layer is used for engaging users in project development through collaboration and content sharing.

There are several roles within the VoRtex ecosystems like students, teachers (authors), developers or content creators, and inspectors. Developers or content creators support the creation of VR content and required features like wallets and payment gateways for end users like students or teachers. Inspectors monitor and submit different high-quality content and learning materials for the community. A student can vote for new content on VoRtex platform using the blockchain network. Teachers are provided with tools for building learning material and lesson scheduling support. Student and teachers are only roles inside VLE. Each role can earn or create content using different approaches, these are:

- Teachers will provide content to the students, and students can buy content using the token provided by the platform. Also, they can sell token to another user and trade token with traditional money

- User can earn token by creating new content (3D model, lectures, etc.)

- Platform activities that include an achievement list that users need to accomplish and earn tokens.

\section{Virtual agents and MicroLessons}

For better user experience, inside VWs we can use chatbots or a virtual agent [25]. Virtual agents recognize the content and understand what it means in a specific context that can be in the form of text, voice, 2D, 3D object, etc. They can utilize information from different sources, like Natural Language Processing (NLP) tools with real-time insights like Microsoft LUIS, IBM Watson, Amazon Lex, Google Dialogflow, and more. Virtual assistants can increase productivity and automate the process, especially in industries like education that rely on communication and handle a set of student issues and questions.

We proposed a solution for developing non-playable characters (NPCs) inside the virtual environment based on machine learning tools (Fig. 4). The intelligent agent component is used to enable multi-sensory communication and the cognitive feature of Professor Vortex NPC that we use for tutoring VLE users. Professor Vortex rewards the user with VRC every time user tag an item in virtual reality that he has not yet recognized. Also, Professor Vortex rewards students with the token if they are active during sessions or have good mark during the semester. Providing data for training of machine learning models user can contribute platform development and AI-based modules, and students can exchange their reward for ETH or another cryptocurrency. 


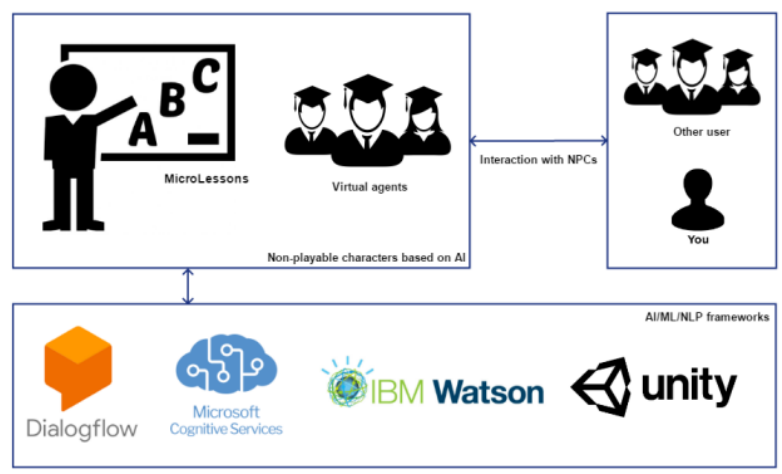

Fig. 4 Intelligent agents software design

Storytelling is a process for driving emotion inside experience, and it is often used in movies and video games. However, we explore a new way of how to embed narration inside WV. VoRtex platform uses MicroLessons concept that includes short educative lectures driven by narrative using the VW environment and NPCs. MicroLessons are small events that are triggered as a result of student interactions, business logic or other factors. Their structure includes the sequencing of events, NPCs, and challenges like solving puzzles for learning new programming algorithm or explore space to find required objects. MicroLessons can occur in isolation or can be called by another event (recursive or sequenced). Also, MicroLessons build a balance between experiences, and improve the interface for collaboration during learning.

Below we will describe a VoRtex prototype for the educational purpose.

\section{E. VoRtex prototype implementation details}

VoRtex prototype has implemented the following features:

- Networking using LAN network (server and clients),

- Communication using the chat system,

- Freedom of movement inside a VW,

- Freedom of choice of avatar representatives in a VW,

- Privileges between user roles,

- Object manipulation over interactable objects in a VW,

- View perspectives (first-person and third-person),

- Public engagement layer (VRC cryptocurrency)

Currently, there are two versions of the Vortex platform, Windows PC and Android Cardboard VR. Regarding the VoRtex Android Cardboard VR version, the prototype is developed using the Unity game engine. The user can control his avatar in the environment by moving his HMD on an object in VW. The Windows version contains more features and better support. Also, we described the Windows version below.

The implemented VoRtex prototype contains two main parts: the client application for desktop personal computers and a back-end server platform. The client application is developed using the Unity game engine. As a backend solution, we use the following tools and technologies: C\#, UNet networking, IBM Watson, Forge Networking, OpenCV 
with Python and C++, MySQL and PHP. Based on the client-server networking system, the teacher creates a host server where students can connect during the lecture as a client. The teacher that hosted the session had a privilege to allow students to manipulate a VW content such as a video and presentation panel, where the users could change the presentation slides that were synchronized with the server and all clients during the session.

For networking system, the platform uses the UNet networking library from a Unity and backend server supported using PHP and MySQL database. Also, PHP and MySQL databases are leading technologies on the backend side of the VoRtex platform. The user can control his avatar and manipulate interactable objects in the environment using a keyboard and a mouse. The VoRtex prototype provides the first-person and third-person view perspectives (Fig. 5 and Fig 6). During lectures, the user can communicate with the teacher or interact with the presentation panel (right side of Fig. 5) and other interactable objects like video panel (left side of Fig. 6). The interactable object can be synchronized among users like the presentation panel where the user can change the presentation slide and propagate this change to all users in the session. The teacher provides authorization for the in-world content to students.

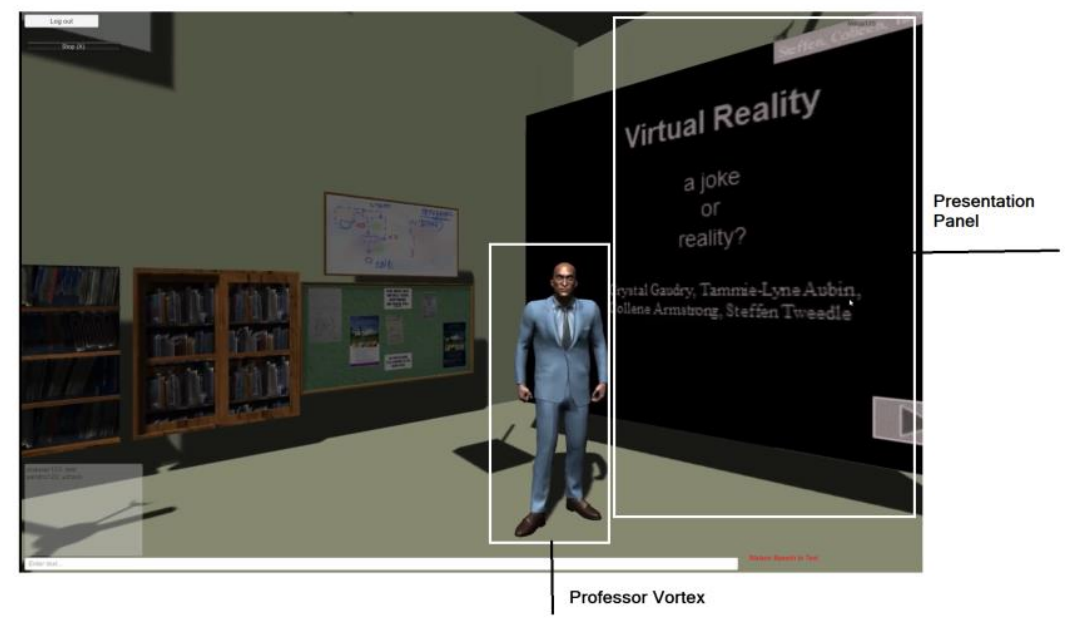

Fig. 5 Professor Vortex inside VLE

Before the users accessed the environment, they had created a login account and chosen avatars that would represent them in a VW. For access system and face recognition, we use a Python and $\mathrm{C}++$ face recognition module with OpenCV, dlib (facial landmarks library) and a deep learning technology stack. The developed module can recognize the user's face with different facial expressions. We integrate this feature into the VoRtex prototype after the user enters the login credentials (username and password) and before he or she accesses the active session. Salts and random data are used to safeguard passwords in the database.

The VoRtex prototype MicroLesson contains a VW classroom with 3D material that supports learning in a real-life environment. The VW classroom contains a table, chairs, a blackboard, projectors, a video and presentation panel and a virtual agent that assists the teacher during lectures. The users can interact with the intelligent agent using voice 
controls. Speech recognition (speech to text) software takes the user voice as input and determines which words were spoken as output that typically consists of a word graph. Based on the generated output, the event executes inside the VW. We use IBM Watson services in the Unity game engine. IBM Watson allows us to transform the client application into the environments where the users and Professor Vortex can interact using text to speech and speech to text services. The users can communicate using the chat system (bottom left in Fig. 6). We implement a chat system using Forge Networking as a separate component with a goal of high reliability and availability of a networking system and in order to avoid a single point of failure. This means that if a session stops, then we can use the chat system to check with other users what measures to take in order to fix the issue.

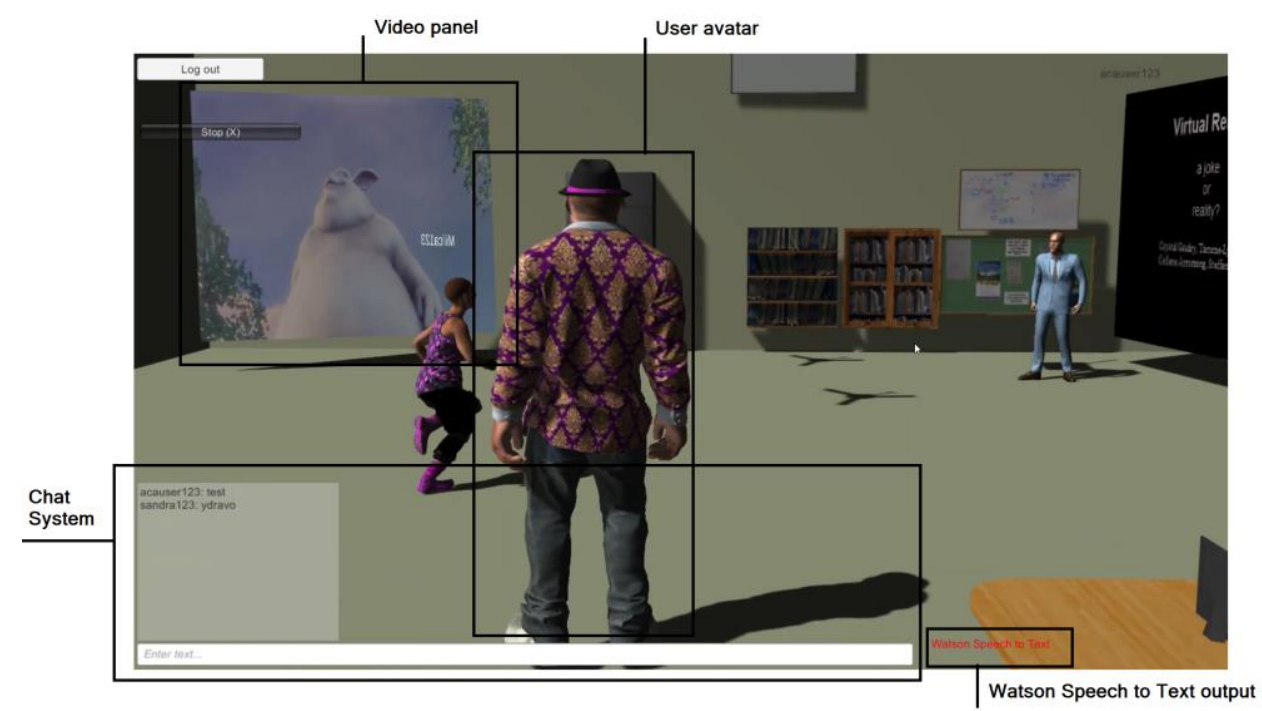

Fig 6. VoRtex virtual environment (Windows desktop, third-person perspective)

\section{F. Public engagement layer implementation details}

We implemented an engagement layer for enterprise features that includes VoRtex Coin (VRC) cryptocurrency based on ERC20 standard and ETH public blockchain technology. The public engagement layer implementation is based on Nethereum package, an open source .NET integration library for Ethereum. Development stack also includes MetaMask for wallets, Remix IDE, Etherscan, and it is tested on the Ethereum Ropsten testnet. In VoRtex platform implemented public engagement layer provides the ability to earn token by engaging the user in lecture activities. The achievement list contains the following activities: manipulate video, manipulate presentation and communicate with a virtual agent. Objects that support mentioned activities we called interactable object. The interactable class diagram is represented in Fig. 7. 


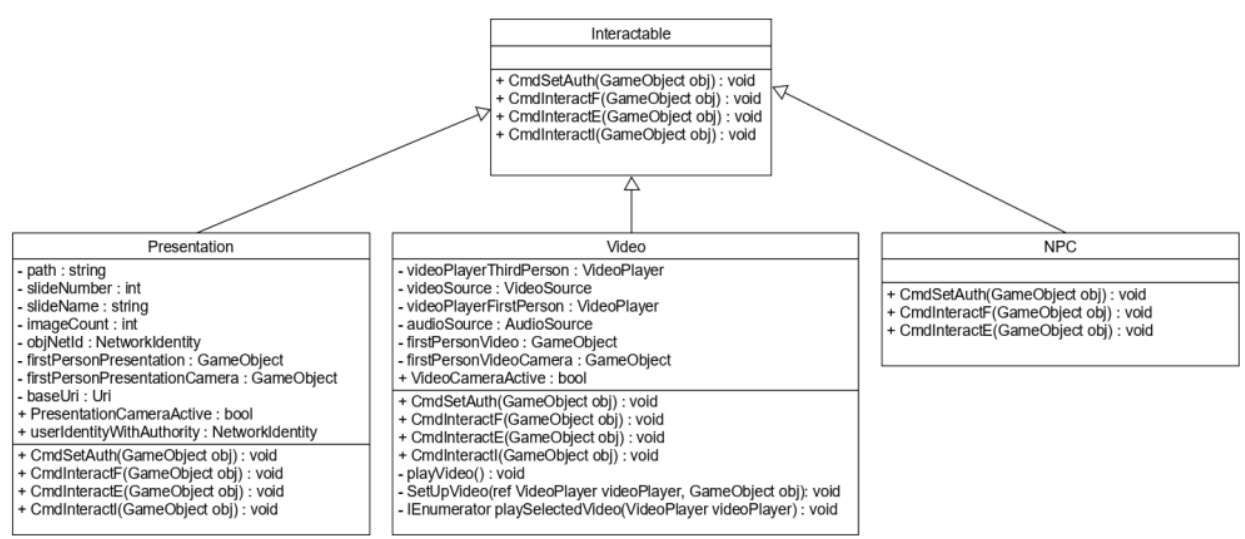

Fig. 7 Interactable class diagram

User wallets are created inside the VoRtex platform. Vortex Coin is currently available only on testnet. User can check VRC wallet status on the top of the application screen and achievement list on the top right side of the application screen (Fig. 8). After executing an action on interactable object token service will validate the action by checking the current achievement list. If the user did not acquire this achievement, then the token service will provide VRC token to the user based on the amount set for that achievement. Use case of the token acquisition process is represented in Fig. 9.

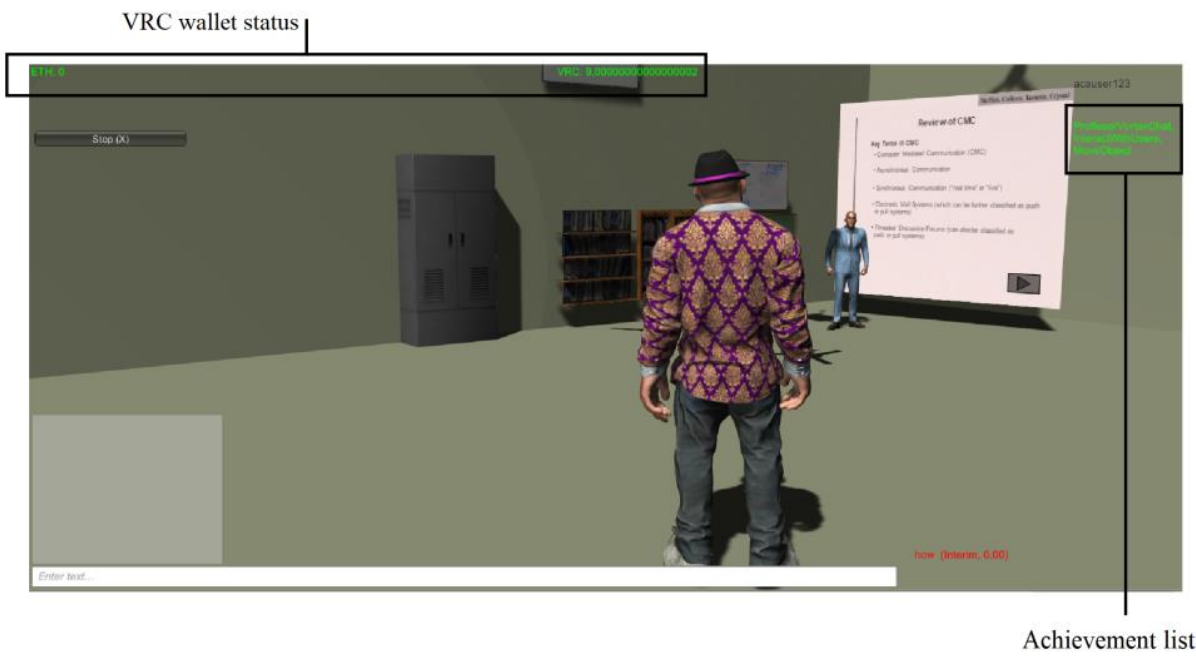

Fig. 8 VoRtex prototype VRC status showcase 


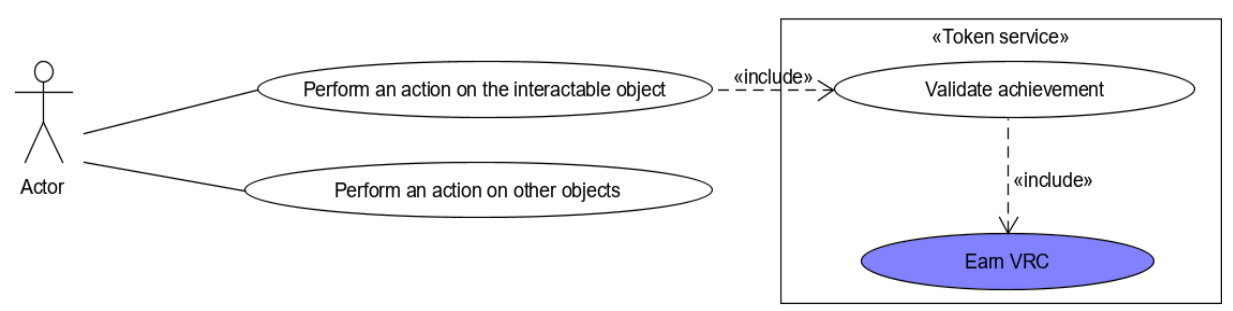

Fig. 9 Token acquisition process use case diagram

\section{EVALUATION AND DISCUSSION}

Based on software architecture evaluation methods [26], we provide a feature table (Table 1) based on software requirements and scenarios standard for B-VR platform development. Also, based on the feature table, we would like to showcase the potential of the VoRtex enterprise solution by marking $(\mathrm{X})$ essential features for each platform.

Table 1 B-VR project comparison

\begin{tabular}{lcccccc}
\hline \multirow{2}{*}{ Criteria } & \multicolumn{5}{c}{ B-VR Virtual Worlds } \\
\cline { 2 - 7 } $\begin{array}{l}\text { VW and avatar } \\
\text { customisation }\end{array}$ & $\mathrm{X}$ & CEEK & Decentraland & Virtual Universe & Vibehub & Mark.Space \\
$\begin{array}{l}360 \\
\text { videos }\end{array}$ & & $\mathrm{X}$ & $\mathrm{X}$ & $\mathrm{X}$ & $\mathrm{X}$ & $\mathrm{X}$ \\
$\begin{array}{l}\text { 3D computer } \\
\text { graphics }\end{array}$ & $\mathrm{X}$ & $\mathrm{X}$ & $\mathrm{X}$ & $\mathrm{X}$ & $\mathrm{X}$ & $\mathrm{X}$ \\
$\begin{array}{l}\text { Open source or } \\
\text { SDK tools }\end{array}$ & $\mathrm{X}$ & $\mathrm{X}$ & $\mathrm{X}$ & & & \\
$\begin{array}{l}\text { AI and } \\
\text { storytelling }\end{array}$ & $\mathrm{X}$ & & & $\mathrm{X}$ & & Only AI \\
$\begin{array}{l}\text { Cryptocurrency } \\
\text { support }\end{array}$ & $\mathrm{X}$ & $\mathrm{X}$ & $\mathrm{X}$ & $\mathrm{X}$ & $\mathrm{X}$ & $\mathrm{X}$ \\
\hline
\end{tabular}

\section{A. Future improvement}

VoRtex platform will require an improved blockchain system in the future. For that reason, we investigate potential technologies that will enhance the current solution and provide a better user experience. One of the solutions is Tendermint [27] Byzantine Fault tolerant state machine replication technology. Tendermint is a partially synchronous BFT consensus protocol derived from the DLS consensus algorithm. The protocol requires a fixed set of validators identified by their public key. Validators attempt to come to a consensus on one block at a time, where a block is a list of transactions. Voting for consensus on a block proceeds in rounds and each round has a proposer, for proposing a block. The validators then vote, in stages, on whether to accept the proposed block or move on to the next round. The proposer for a round is chosen deterministically from the ordered list of validators, in proportion to their priority. Users can update the global state 
through transactions made in VoRtex blockchain or through off-chain VR channels (Fig. 10). ChainLink [28] is a secure blockchain middleware that allows Ethereum SMs on the platform to connect to external, off-chain resources through external data feed triggers. By creating a decentralized infrastructure for SMs to access critical off-chain resources, it will create a scalable and secure way to add data. VoRtex platform will use a traditional database sharding technique, which divides a database into samples and distribute them on a different server. Then, each node stores a subset of data and only verifies selected transactions. Also, the node can find transactions information from another node.

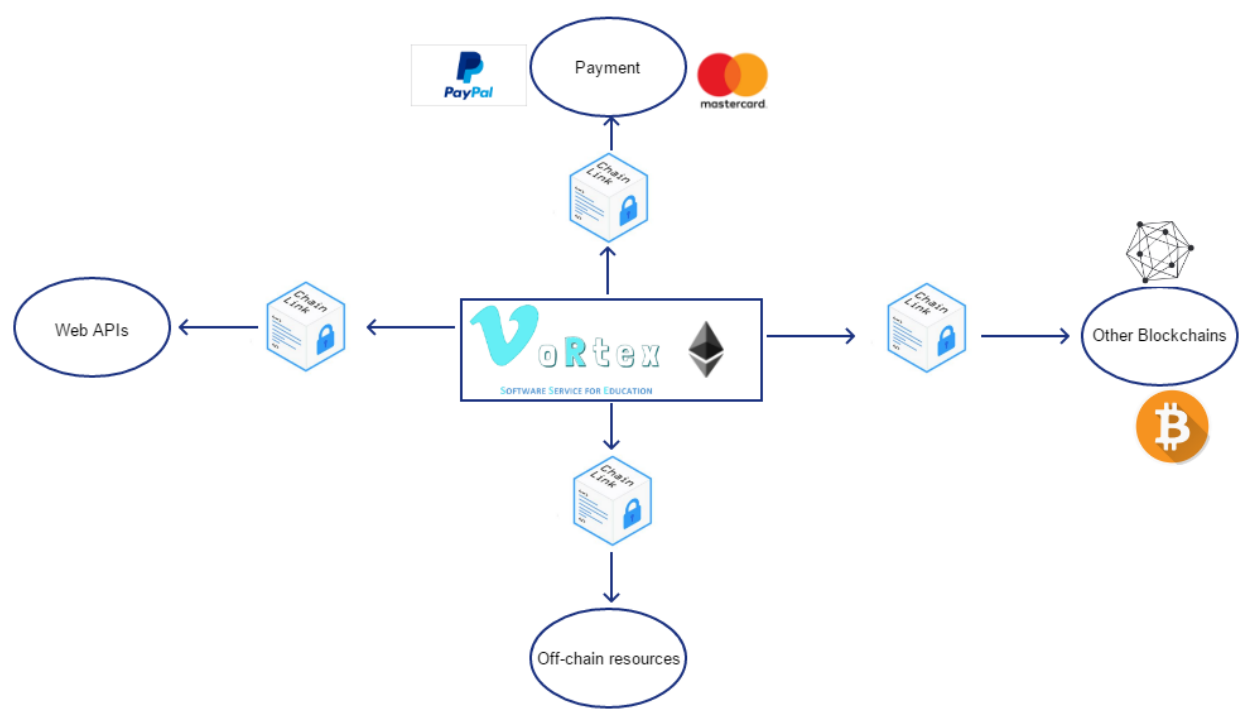

Fig. 10 Decentralized infrastructure for SMs

VoRtex will allow teachers and authors to create virtual items using a tool called Coin Mint for creating custom minted coins or their currencies which are used for as revenue streams. Items produced using the Coin Mint tool can be used for purposes such as conference tickets in a virtual environment or for assistive items that provide a better user experience. Teachers or authors will sign these items with unique cryptographic token signatures that cannot be duplicated. The VoRtex Virtual Mint Registry will keep track of created coins, and other virtual items which will be recorded onto the blockchain for immutable tracking. Each coin or item will be ERC20 compliant and be provided with their own unique Ethereum address through a type of ERC20 adapter that uses the Bancor Protocol. Payment gateway through the support of SM and ChainLink will govern the onchain and off-chain payments. The smart wallet will allow users to store their valuable, virtual items, custom tokens, coins, courses, custom items and anything of value in VW.

\section{$B$. Scalability and security issues}

For scalability, VR systems have no established backup system for data due to the enormous amount of computing and graphics power needed to render virtual environments. 
Content distribution across a P2P network is challenging because of slow download speeds and integrity of content distributed around the system. However, blockchain solution like Filecoin [29] protocol is addressing these issues. Using Filecoin network, anyone can participate as storage providers. Also, a persistent and continuously updated collection of virtual spaces could decrease the computational costs for VW applications and expand the available interactive scope. However, from a performance perspective, traditional VR platforms with the centralized network will initially beat blockchain-based platforms in content distribution speed, while blockchain-based solutions will have an advantage through its virtual marketplace for buying content with tokens that are tradable for traditional currency.

Regarding robustness and security, we should provide better error handling during a transaction between token transactions. ERC20 standard uses two ways for a token transaction using transfer, or the combination approves and transferFrom functions. The transfer function lets the user send tokens to address. However, if the user wants to deposit tokens to an SM, then use the combination approve and transferFrom functions. The user should authorize SM to withdraw tokens via the approve function. Afterwards, call service of an SM that will handle deposits and revoke tokens via the transferFrom function. If the blockchain developer uses the transfer function to deposit tokens to an $\mathrm{SM}$, then the transaction will succeed, but will not be accepted by the acceptor contract. Also, if the exchange contract does not implement an emergency token extraction function, then it will result in a permanent loss of the tokens. The bug of the ERC20 token SM involves hundreds of millions of dollars. Similar issues have arisen in another public blockchain that supports SMs, but they have not received much attention [30]. Modifying the code of the SM, the vulnerability cannot be fixed, even if the source code affected by the error only involves the programming logic that does not include the cryptocurrency, developers cannot do anything about this issue. Usually, whenever a mistake like this happens in a traditional application, then it will crash, and the user will lose data. However, if an error occurred in SM, then data can either be dropped or locked up without any change of retrieval. Most software developers are not aware of ERC223 and ERC777 standard. The ERC223 standard focuses on security and resolves the ERC20 critical bug by making the transfer function throw an exception on invalid transfers and cancelling the transaction, without losing the data. The ERC777 standard focuses on mainstream adoption and solves ERC20 issues, such as the need for transaction handling functionality.

\section{CONCLUSION}

In this paper, we introduced blockchain-based VW projects. Firstly, we reviewed related work regarding VW and blockchain. Also, we introduced scientific and commercial projects in this field. Secondly, we described the concept and purpose of the blockchain-based solution in VWs. Thirdly, we introduced VoRtex enterprise decentralized VR blockchainbased software architecture to support the development of education, certification and MOOC. We described the components and technologies that will supply our decentralized project. Afterwards, we showcased practical examples inside VoRtex platform using cryptocurrency to engage in MicroLesson activities. Then, we provided a feature table that contains a comparison between B-VR solutions and VoRtex platform. In the end, we provide guidelines for further improvements. 
In our future work, we will improve evaluation methods for blockchain-based VW architecture. Blockchains and open source software could revive the potential of VR for education and research. Blockchain technology can enhance the mechanism for calculating scholarships or salaries for student or teachers with a transparent system for funding projects. For our prototype, we choose the ETH blockchain platform because of popularity among the community and available framework for rapid prototype development. However, solutions like NEO, EOS or Disciplina could be added as a replacement in future versions of platforms. We should mention that the VoRtex prototype is functional with described features like biometric verification, user registration, networking, chat system, teacher and student authorization during MicroLesson, object interaction, etc. Demo showcase can be requested from a VoRtex website [23] and all implemented features can be found on VoRtex YouTube channel [31]. Also, the VoRtex blockchain component will be tested with active users and then published on Mainnet.

\section{REFERENCES}

[1] Aleksandar Jovanović and Aleksandar Milosavljević, Review of Modern Virtual Reality HMD Devices and Development Tools, ICEST 2017 Conference. 52nd International Scientific Conference on Information, Communication and Energy Systems and Technologies - Serbia, Niš, June 28 - 30, 2017.

[2] Kim J. L. Nevelsteen, "Virtual world, defined from a technological perspective and applied to video games, mixed reality, and the Metaverse", Computer Animation and Virtual Worlds, Volume 29, Issue 1 January/February 2018

[3] F. J. García-Peñalvo, J. Cruz-Benito, O. Borrás-Gené, and Á. Fidalgo Blanco, "Evolution of the Conversation and Knowledge Acquisition in Social Networks Related to a MOOC Course," in Learning and Collaboration Technologies. vol. 9192, P. Zaphiris and A. Ioannou, Eds., ed: Springer International Publishing, 2015, pp. 470-481

[4] Xueping Liang, Juan Zhao, Sachin Shetty, Jihong Liu, Danyi Li, "Integrating Blockchain for Data Sharing and Collaboration in Mobile Healthcare Applications," 2017 IEEE 28th Annual International Symposium on Personal, Indoor, and Mobile Radio Communications (PIMRC)

[5] Hyungeun Jo and Sungjae Hwang. 2013. Chili: viewpoint control and on-video drawing for mobile video calls. In CHI'13 Extended Abstracts on Human Factors in Computing Systems. ACM, 1425-1430.

[6] Bektur Ryskeldiev, Yoichi Ochiai, Michael Cohen, and Jens Herder. 2018. Distributed Metaverse: Creating Decentralized Blockchain-based Model for Peer-to-peer Sharing of Virtual Spaces for Mixed Reality Applications. In Proceedings of the 9th Augmented Human International Conference (AH '18). ACM, New York, NY, USA, Article 39, 3 pages. DOI: https://doi.org/10.1145/3174910.3174952.

[7] S. Rueda, P. Morillo and J. M. Orduna, A comparative study of awareness methods for peer-to-peer distributed virtual environments, Computer Animation and Virtual Worlds, Volume 19, Issue 5 December 2008

[8] Mingfei Wang, Jinyuan Jia, Ning Xie and Chenxi Zhang, Interest-driven avatar neighbor-organizing for P2P transmission in distributed Virtual Worlds, Computer Animation and Virtual Worlds, Volume 27, Issue 6 November/December 2016

[9] Theodore Kotsilieris, George T. Karetsos, Ioannis Anagnostopoulos and Nikoletta A. Dimopoulou, Interconnecting distributed Virtual Worlds using Metabots performance evaluation against the traditional client-server model, Computer Animation and Virtual Worlds, Volume 26, Issue 6 November/December 2015

[10] Grech, A. and Camilleri, A. F. (2017) Blockchain in Education. Inamorato dos Santos, A. (ed.) EUR 28778 EN; doi:10.2760/60649

[11] Antonio Bartolome, Carles Bellver Torla, Linda Castaneda, Jordi Adell, Blockchain in education: introduction and critical review of the state of the art. doi: 10.21556/edutec.2017.61

[12] Turkanovic, M.; Holbl, M.; Kosic, K.; Hericko, M.; Kamisalic, A. EduCTX: A blockchain-based higher education credit platform. IEEE Access 2018, doi:10.1109/ACCESS.2018.2789929.

[13] All-in-One Blockchain Solutions. Available on: https://ark.io/, accessed Jun 24, 2019. 
[14] Disciplina. Available on: https://disciplina.io/, accessed Jun 24, 2019

[15] Kadam, Suvarna. (2018). Review of Distributed Ledgers: The technological Advances behind cryptocurrency

[16] Satoshi Nakamoto. 2009. Bitcoin: A Peer-to-Peer Electronic Cash System. (2009). https://bitcoin.org/bitcoin.pdf, accessed Jun 24, 2019.

[17] Prasad Satyavolu and Abhijeet Sangamnerka. 2016. Blockchain's Smart Contracts: Driving the Next Wave of Innovation Across Manufacturing Value Chains. (June 2016). Cognizant 20-20 Insights, https://www.cognizant.com/whitepapers/blockchains-smart-contracts-driving-the-next-wave-ofinnovation-across-manufacturing-value-chains-codex2113.pdf, accessed Jun 24, 2019.

[18] Ethereum Github Site 2017. A Next-Generation Smart Contract and Decentralized Application Platform. (2017). https://github.com/ethereum/wiki/wiki/White-Paper, Jun 24, 2019.

[19] ERC20. Available on: https://theethereum.wiki/w/index.php/ERC20_Token_Standard, accessed Jun 24, 2019.

[20] CEEK. Available on: https://www.ceek.io/, accessed Jun 24, 2019.

[21] Esteban Ordano, Ariel Meilich, Yemel Jardi, and Manuel Araoz. 2017. Decentraland: A blockchainbased virtual world. https://decentraland.org/whitepaper.pdf, accessed Jun 24, 2019.

[22] VibeHub. Available on: https://vibehub.io/, accessed Jun 24, 2019.

[23] VoRtex. Available on: http://www.vortexsoftwareservice.com/, accessed Jun 24, 2019.

[24] Don Tapscott and Alex Tapscott, The Blockchain Revolution and Higher Education. Available on: https://er.educause.edu/articles/2017/3/the-blockchain-revolution-and-higher-education, accessed February 24, 2019.

[25] Kang, Yilin \& Tan, Ah-Hwee. (2013). Self-Organizing Cognitive Models for Virtual Agents. 8108. 10.1007/978-3-642-40415-3_3.

[26] Gampa, Shreelekhya \&, Yazhini \& Senthilkumaran, U \& Manikandan, N. (2016). Methods for evaluating software architecture-A survey. International Journal of Pharmacy and Technology. 8. 25720-25733.

[27] Tendermint. Available on: https://tendermint.com/, accessed Jun 24, 2019.

[28] Chainlink. Available on: https://chain.link/, accessed Jun 24, 2019.

[29] Filecoin. Available on: https://filecoin.io/, accessed Jun 24, 2019.

[30] J. Bonneau, A. Miller, J. Clark, A. Narayanan, J. A. Kroll, and E. W. Felten. 2015. SoK: Research Perspectives and Challenges for Bitcoin and Cryptocurrencies. In Proc. IEEE Symp. Security and Privacy.

[31] VoRtex YouTube channel. Available on: https://www.youtube.com/channel/UC_JffLKo1rZQWKra5Arh6ag, accessed Jun 24, 2019. 